\title{
Más allá de peregrinos y de oro: desarrollo social precolombino Costa Rica, Panamá y el norte de Colombia
}

\author{
Mauricio Murillo Herrera*
}

\begin{abstract}
Murillo Herrera, Mauricio. Más allá de peregrinos y de oro: desarrollo social precolom-
\end{abstract} bino Costa Rica, Panamá y el norte de Colombia. R. Museu Arq. Etn., 31: 56-79, 2018.

Resumen: Se expone de manera integral los principales avances en la comprensión del desarrollo de las sociedades precolombinas que habitaron en el territorio que hoy se conoce como Costa Rica, Panamá y el norte de Colombia y se realiza una valoración crítica de los resultados; esto con el fin de aumentar nuestra comprensión acerca de los procesos de cambio social precolombino en la región, principalmente aquellas que se han realizado en las últimas dos décadas. El análisis se realiza tanto para desarrollos sociales tempranos como para las sociedades tardías, y éstas últimas se estudian a partir de las temáticas propuestas por los investigadores como los principales impulsores de cambio social. El artículo muestra la extraordinaria diversidad en adaptaciones y procesos de desarrollo social ocurridos en la región durante la época precolombina, lo cual va en contra de una idea de homogeneidad social y cultural. Por otra parte, se destaca que efectivamente ha habido avances en el estudio de las sociedades antiguas en la región, pero estos no han sido aún mayores debido a que 1) se ha confundido elaboración conceptual con aumento en la comprensión de los desarrollos sociales y porque 2) no se ha logrado superar la brecha epistemológica y metodológica que existe entre describir el material y el contexto arqueológico y estudiar procesos sociales en el pasado. Finalmente, se muestra que si se quiere entender todo el rango de desarrollos sociales precolombinos que ha sido descrito en esos tres países, es necesario considerar dentro de los modelos otros factores de cambio distintos a los que han sido históricamente propuestos para la región: intercambio e interacción interregional.

Palabras clave: Arqueología; Cambio social; Colombia; Panamá; Costa Rica.

\section{Introducción}

F $\mathrm{n}$ el sur de América Central y el norte de América del Sur se desarrollaron sociedades autóctonas que se organizaron de forma distinta al Estado, en unidades sociales menores que, prácticamente, se

\footnotetext{
* Profesor e investigador, Escuela de Antropología. Centro de Investigaciones Antropológicas. Universidad de Costa Rica.<mauricio.murilloherrera@ucr.ac.cr>
}

encuentran extintas hoy día. A lo largo del último milenio, solo un minúsculo puñado de formas sociales ha sobrevivido ante el avance irrefrenable de estados e imperios. La importancia de esta región del mundo, en el estudio de sociedades antiguas, se destaca aún más, si se considera que dichas sociedades se desarrollaron en medio de dos grandes núcleos de sociedades estatales que surgieron en México, Guatemala y en el norte de Honduras y en Bolivia, Perú y en el sur de 
Ecuador. A pesar del contacto que ha sido demostrado, de las posibles incursiones civiles y militares provenientes de estos estados, de las sociedades del sur de América Central y del norte de América del Sur se mantuvieron al margen de dichos desarrollos sociales vecinos, desde una perspectiva sociopolítica. Por lo tanto, el principal aporte que la arqueología, de esta región del mundo, le puede ofrecer al estudio del desarrollo de sociedades antiguas prehistóricas, es proporcionar evidencia en cuanto a los múltiples desarrollos sociales que han sido detectados, exponiendo tanto las diferencias como las similitudes que han sido encontradas. De esta forma, se pueden evaluar progresivamente las distintas hipótesis que están relacionadas con los procesos de cambio social (Kintigh et al. 2014 Smith 2011), así como la evidencia disponible y los avances teórico-metodológicos de los últimos años.
Un único artículo no puede tener la pretensión de abarcar y discutir, ni siquiera de manera superficial, todo el trabajo arqueológico que ha sido realizado en las últimas décadas en los territorios que son conocidos, actualmente, como Costa Rica, Nicaragua y el norte de Colombia, entendiéndose, en este último caso, a la Región de la Costa Atlántica (la cual cubre, aproximadamente, los departamentos de Guajira, Magdalena, Atlántico, César, Bolívar, Sucre, Córdoba) (ver Figura 1). No obstante, únicamente, se discutirá un número limitado de investigaciones que fueron realizadas en algunas regiones del área definida. Probablemente, algunas investigaciones importantes no sean discutidas o mencionadas, sin embargo, las limitaciones de espacio, propias de un artículo, nos fuerza a restringir la discusión a una muestra de casos.

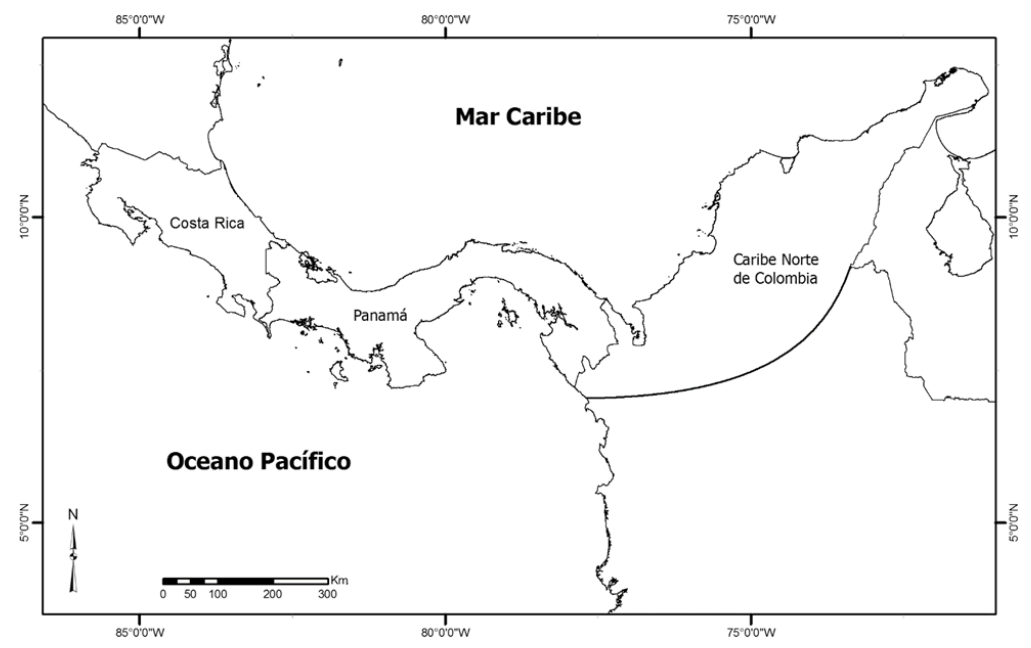

Fig. 1. Región aproximada donde se ubican las distintas regiones a las que se hace referencia en el texto.

\section{Sociedades tempranas (pre-1000 A.C.)}

La presencia de grupos humanos cazadores de megafauna se ha sugerido, a partir de fechas tan tempranas como el 14000 a.C, para el Valle del Magdalena en Colombia ${ }^{1}$ (Van der Hammen \&

1 Aunque hay que señalar que aún se debate la asociación estratigráfica entre artefactos y restos faunísticos (Aceituno Bocanegra \& Rojas Mora 2012: 128)
Correal 2001) mientras que la reciente excavación de un campamento y taller lítico en el sitio La Isla, ubicado en Siquirres, en el sector Caribe de Costa Rica, indican que la presencia humana, en ese sector, podría remontarse al 10000 a.C. ${ }^{2}$ (Chávez

2 Según el análisis de una datación de $14 \mathrm{C}$ que fue realizada a material orgánico (carbón y madera), fue asociado a material lítico (una punta, lascas, raederas, raspadores, preformas). 
Montoya \& Naranjo Masís 2013). En épocas más tardías, hacia finales de la última glaciación, es decir, 8000 a.C., parece haber existido una continuidad en la ocupación humana de las llanuras del Pacífico Central y el pie de monte de la cordillera central de Panamá (Cooke 2005), continuidad que también podría ser descrita para el norte y para el centro de Costa Rica, aunque, allí, la información, al respecto, aún es ínfima. Cooke (2005: 138-140) ha afirmado que si bien se ha mejorado respecto al control temporal para el lapso cuando el ser humano entró al istmo Centroamericano y lo colonizó, este mejoramiento ha sido demasiado lento y el registro sigue siendo demasiado imperfecto como para evaluar hipótesis alternativas que están relacionadas con estos hechos. A pesar de ello, la comparación de la evidencia que estaba disponible tanto del istmo como del norte de Colombia ha llevado a distintos investigadores (Aceituno Bocanegra \& Rojas Mora 2012: 134; Ranere \& López Castaño 2007: 30;) a postular que, para este periodo, existió una economía de amplio espectro que era ligada a una gran diversidad de adaptaciones a los distintos ecosistemas, lo cual desembocó en una divergencia cultural que puede ser rastreada, incluso a partir del Pleistoceno Tardío. Después del 7000 a.C., dichas poblaciones comenzaron a incorporar, paulatinamente, plantas que eran cultivadas a su dieta.

Alrededor del 6000 a.C. varias especies domesticadas de raíces y semillas, como el maíz (Zea mays L.), la yuca (Manihot esculenta Crantz), la maranta (Maranta arundinacea L.) y el ñame (Dioscorea sp.), estaban siendo cultivadas en Panamá, a través de una agricultura de roza y quema (Dickau 2010; Piperno, Bush \& Colinvaux 1991; Piperno et al. 2000). Este proceso inicia en la región Central de Panamá y se difunde hacia Chiriquí, alrededor del 5000 a.C. (Dickau 2010: 128). No obstante, parece ser que, en esta región, la agricultura de quema y roza solo ocurrió cuatro mil años después. Recientemente, Griggs (2005) ha propuesto un modelo para el Caribe panameño con el que postula que su colonización comenzó alrededor del 5000 a.C. y que una presión sobre los recursos de la vertiente Pacífica motivó el traslado de poblaciones desde el Pacífico hacia el centro de la cuenca del Caribe. En Costa Rica, el maíz estaba siendo cultivado en el noroeste del país, alrededor del 4000 a.C. (Arford \& Horn 2004; Bradley 1994), un cultivo intensivo y extensivo del mismo no se registra para el sector este del país, sino hasta alrededor del 2000 a.C. (Lane, Horn \& Mora 2004). No obstante, ni en América Central ni en Colombia parece existir evidencia alguna de que el maíz fuera parte substancial de la dieta de las poblaciones precolombinas antes del 500 a.C.

No es hasta después del 5000 a.C. que se encuentra evidencia de una conducta sedentaria en algunas regiones del istmo. Scott Raymond (1998: 18, 2008) ha señalado que, si bien la explotación de recursos marítimos fue importante económicamente, inicialmente las poblaciones ubicadas en zonas costeras de Colombia y Panamá se proveían, principalmente, de recursos provenientes de ambientes sabaneros, fluviales y boscosos. En Panamá, la pesca en la costa y en los estuarios se intensificó alrededor del 2500 a.C. y parece haber estado ligada a un incremento en el tamaño de los asentamientos y, probablemente, a un modo de vida sedentario, los primeros agricultores se dispersaron desde núcleos de poblaciones anteriores ubicadas en las tierras áridas de la vertiente del Pacífico rumbo a bosques más húmedos, entre 5000 y 2000 a.C. (Cooke 2005: 146).

En el norte de Colombia, hacia el 4000 a.C., emerge una tradición litoral en los planos aluviales de las tierras bajas de la costa Caribe (Aceituno Bocanegra \& Rojas Mora 2012: 126-127) cuya estrategia adaptativa estaba orientada hacia un acceso estacional a diferentes ecosistemas como estuarios, playas, pequeños ríos, lagunas, bosques secos y sabanas. Muchos de estos asentamientos son concheros costeros o ribereños, con cerámica y una tecnología lítica que está relacionada con el procesamiento de recursos vegetales y este patrón económico (Scott Raymond, 1998: 18) continuó a través del primer milenio a.C. La presencia de una economía de subsistencia de amplio espectro, enfocada tanto hacia los recursos marítimos como hacia los terrestres se ha detectado en la costa Caribe de Costa Rica, 
en el sitio Black Creek, un campamento costero habitado alrededor del 3000 a.C. por un grupo humano pequeño alfarero (Baldi 2001, 2011; Chávez, Fonseca \& Baldi 1997).

En tanto que para el 4000 a.C., las poblaciones ubicadas tierra adentro cosechaban semillas salvajes, anticipando así la horticultura junto con el manejo y el cuidado de plantas. Para el caso del sitio San Jacinto I Oyuela-Caycedo \& Bonzani (2005), han propuesto un modelo de movilidad logística como la estrategia de subsistencia más probable. Según este modelo, habría existido un campamento base que ha sido situado estratégicamente, así como otros campamentos para propósitos especiales, todos asociados con un territorio de caza y recolección, el cual pertenecía, exclusivamente, a la población local. Para 3000 a.C., un patrón de menor movilidad pudo haber evolucionado, quizás como resultado de una mayor competencia por los recursos. Alrededor del 2000 a.C., asentamientos aldeanos comienzan a surgir en el norte de Colombia, como lo evidencia, por ejemplo, el sitio San Jacinto II; poco después, entre el 1500 y el 800 a.C., surgen aldeas de hasta $0.5 \mathrm{~km}^{2}$ en el Bajo Magdalena, al mismo tiempo que se detecta un gran aumento de la población (Langebaek \& Dever 2000). Esta última etapa de consolidación de un modo de vida aldeano ya había sido identificada, para el caso Ciénega Grande, en la aldea prehispánica Momil, donde además también se detectó una transición entre una dieta basada en tubérculos hacia un consumo más prominente de semillas (Reichel-Dolmatoff 1989: 39-40, 1997: 97-98).

En su comparación de casos de estudio en Panamá, Colombia y Ecuador, Scott Raymond (2008: 87) no logró identificar una causa común para el desarrollo del sedentarismo. No obstante, argumentó que "el aumento en el tamaño y en la densidad de la población probablemente jugó, al menos en parte, un papel causal en la sedentarización de las comunidades." No obstante, la evidencia regional en el Bajo Magdalena indica que en la región hubo una densidad de población muy baja, previo a la adopción de la vida sedentaria, solo después de este evento el tamaño de la población se dispara (Langebaek \& Dever 2000: 52).

En resumen, conforme avanza el conocimiento respecto a los procesos de sedentarización y adopción de la agricultura en las poblaciones de la región, resulta cada vez más difícil generalizar en torno a ellos. Ciertamente, la evidencia por ahora nos indica que, entre los grupos humanos antiguos ubicados entre Costa Rica y el norte de Colombia, la interacción entre humanos y plantas tuvo lugar desde comienzos del Holoceno y, además la domesticación de plantas precedió por alrededor de 4000 años la sedentarización definitiva de estas poblaciones (Archila Montañez 2008: 77, 82), no obstante, según la evidencia arqueológica disponible, los desarrollos sociales entre el 5000 y el 1000 a.C. fueron múltiples y muy variados (Aceituno Bocanegra \& Rojas Mora 2012: 146; Llanos Vargas 1993: 47). Así mismo, los argumentos difusionistas en torno a la adopción de la agricultura en esta región del mundo se ven cada vez menos apoyados por la evidencia acumulada (Archila Montañez 2008; Langebaek \& Dever 2000: 52). Resulta interesante, por lo tanto, que la evidencia lingüística y genética parece contar otra historia, como más adelante se discutirá. Lo anterior advierte sobre el peligro de homologar distintos aspectos de la vida social (lenguaje, genética, cultura material) que no tienen que coincidir, sino, al mismo tiempo, motiva a investigar, por ejemplo, por qué no coincidieron en esta región del mundo.

Por otra parte, los datos que fueron mencionados indican que en las décadas recientes ha habido avances en la investigación sobre sociedades de cazadores-recolectores, domesticación de plantas y procesos de adopción de vida sedentaria en Panamá (e.g. Cooke 1992, 1995, 2005; Cooke \& Ranere 1992; Hansell 1987; Ranere \& Cooke 1996; Ranere \& Cooke 2003; Ranere \& Hansell 1978) y en la región de la Costa Caribe de Colombia (e.g. Aceituno Bocanegra \& Rojas Mora 2012; Langebaek \& Dever 2000; OyuelaCaycedo 1987c, 1993, 1996; Oyuela-Caycedo \& Bonzani 2005; Ranere \& López 2007; Scott Raymond, 1998, 2008; Reichel-Dolmatoff 1997; 
Van der Hammen \& Correal 2001). Mientras que, para el caso de Costa Rica, la investigación que está relacionada a estos procesos tempranos continúa avanzando muy lentamente y aún depende de hallazgos esporádicos. Desde los hallazgos del Valle del Turrialba (Snarskis 1977, 1979) hasta el reciente hallazgo de La Isla en Siquirres (que fue comentado anteriormente), que se obtiene nueva información acerca de este periodo. Para el Formativo, en los últimos veinte años ha habido también algún avance para Costa Rica con el estudio de Black Creek, el cual también ya comentamos y del sitio Los Sueños, una pequeña aldea ubicada en el Pacífico Central, poblada en algún momento del lapso comprendido entre 1500 y 300 a.C. (Corrales Ulloa 2000, 2006). Actualmente, este periodo está también siendo investigado en Siquirres, en el sector Caribe (Chávez Montoya \& Naranjo Masís 2013).

\section{Desarrollos sociales tardíos (1000 a.C.-1550 d.C.)}

Un repaso somero por la literatura de la región hace evidente que en la investigación en el norte de Colombia, Panamá y Costa Rica siga predominando temáticas como las secuencias cronológicas, la cerámica, el intercambio de bienes, la arquitectura monumental y el análisis y la descripción de artefactos excepcionales, ya sea por su estética o por su exotismo (Angulo Valdés 1978, 1983, 1987; Bray 1984, Cadavid Camargo \& Groot de Mahecha 1987; Cooke 2005; Corrales Ulloa 2000; Fonseca Zamora 1997a; Fonseca Zamora \& Cooke 1994; Giraldo Peláez 2010; Groot de Mahecha 1980, 1989; Hoopes 2005; Langebaek 1987a; Langebaek \& Cárdenas Arroyo 1996; Lleras Pérez 1987; Quilter \& Hoopes 2003; Reichel-Dolmatoff 1997; Serje de la Ossa 1987; Snarskis 2003; Uribe Tobón 1988; Uribe Villegas \& Martinón-Torres 2014;

Valderrama Andrade \& Fonseca Truque 1981).

No obstante, en los últimos años ha habido, también, avances significativos en el estudio de instituciones sociales y del cambio social en distintas regiones del sur de América Central y del norte Colombia. Estos estudios han dado luces en torno a no solo los factores implicados en el cambio, sino también en cuanto a los mecanismos detrás de los procesos de desarrollo de sociedades precolombinas. Estudios anteriores (Drennan 1995, 1996) han dado cuenta de los avances que se han logrado en torno a la comprensión de las funciones que pudieron tener distintos factores (e.g. intercambio, presión demográfica, guerra, intensificación agrícola), dentro de los procesos de cambio social precolombino en el sur de América Central y en el norte de América del Sur. A continuación, se verá cómo algunas investigaciones recientes en el norte de Colombia, Panamá y Costa Rica (el territorio más central del área geográfica que fue mencionada arriba) han dado nuevas luces en torno a la función diferencial de estos factores sobre los procesos de desarrollo social en la región.

\section{Relaciones interregionales a larga distancia}

Al hacer referencia al surgimiento de sociedades cacicales en el sur de América Central y el norte de América del Sur es inevitable mencionar los contactos o las relaciones interregionales entre estas dos regiones, tema que, históricamente, ha dominado la literatura de la arqueología de este sector del planeta. El vínculo América Central-América del Sur ha sido destacado, por diversos investigadores (Kidder 1940; Lothrop 1940; Fonseca Zamora \& Richardson 1978; Willey 1971), a lo largo del siglo veinte. Las bases arqueológicas de esta relación han sido, principalmente, similitudes estilísticas y formales en los artefactos que fueron encontrados (Bray 1978, 1984, 1992, 1997, 2003; Cooke 1998, Falchetti 1979, 1987, 1993; Hoopes 2004, 2005, 2011; Sáenz Samper \& Lleras Pérez 1999; Sánchez Herrera \& Cooke, 1997; Uribe Villegas 1988), o bien la presencia de una arquitectura monumental similar (Fonseca Zamora 1981; Hoopes 2011; Snarskis 2003: 187; Reichel-Dolmatoff 1965, 1990, 1997), en sitios como Pueblito y Buritaca 200, en el norte de Colombia, Guayabo de Turrialba 
y otros sitios en el centro y este de Costa Rica. Dichas similitudes han sido explicadas como producto de interacciones interregionales entre grupos del centro y del sur de América, ya sea por tierra (Correal Urrego 1990: 84; Cooke 1998; Fonseca Zamora 1997b; Helms 1979; Mason 1931; Reichel-Dolmatoff 1965, 1983: 95; Reyes Paniagua 2009a, 2009b) o a través de viajes marítimos (Callaghan \& Bray 2007; Fonseca Zamora \& Richardson 1978).

En las últimas décadas, esta relación se ha delimitado y se ha focalizado regionalmente entre Costa Rica, Panamá y el norte de Colombia (Bray 1984, 2003; Cooke 1992, 2005; Fonseca Zamora 1997a; Helms 1979; Hoopes 2004, 2005, 2011; Quilter \& Hoopes 2003). Por ejemplo, Helms (1979) utilizó, principalmente, relatos etnográficos cuando afirmó que las relaciones a larga distancia entre jefes tribales de Panamá y líderes de cacicazgos, ubicados en Colombia, fueron el elemento clave para el surgimiento de cacicazgos complejos en el centro de Panamá. Según la autora, el conocimiento esotérico y los bienes de prestigio que representan poder, tales como ornamentos de oro, fueron los recursos que estos jefes obtuvieron en los Andes colombianos. Sin embargo, investigaciones arqueológicas recientes (Haller 2008; Isaza Aizpurúa 2007), las cuales han utilizado otro tipo de evidencia tal como patrones de asentamientos, cálculo de densidades de materiales, tamaño de asentamientos, uso diferencial de áreas de actividad humana y otras, apuntan a que el surgimiento de la complejidad social en el centro de Panamá parece no haber tenido relación alguna con la ostentación de bienes de prestigio. Más bien parece ser que la explotación de recursos marítimos y agrícolas locales, así como con el surgimiento de redes de intercambios locales y regionales de estos productos fueron elementos determinantes en los procesos de desarrollo social. Además, a pesar de que ciertamente la metalurgia fue introducida en el sur de América Central a través de Colombia (Bray 1984, 1992, 1997), para el momento en que se detecta el surgimiento de complejidad sociopolítica en el centro de Panamá ( 700 d.C.), el oro ya era obtenido y era producido a nivel local (Cooke et al. 2003). Esto implicaría que los jefes de Panamá no tuvieron necesidad de buscar emblemas de poder, como el oro (ya sea en su materia prima o en condición de artefacto), en territorios lejanos.

Para el caso de las sociedades antiguas, que estuvieron ubicadas en el territorio actual de Costa Rica, el surgimiento de jerarquía social institucionalizada se ha relacionado con la presencia de artefactos provenientes de Mesoamérica y de un vínculo ideológico de dichos objetos con un culto al maíz, con la intensificación de la agricultura como producto de la adopción del maíz, con un crecimiento demográfico inmediato y con el surgimiento de sociedades cacicales (Corrales Ulloa 2001; Fonseca Zamora 1992; Snarskis 1981, 1984, 1986, 2003). De acuerdo con Snarskis, después de 500 d.C., la caída de Teotihuacan hizo que se cortaran las rutas comerciales entre Mesoamérica y el sur de América Central, lo que ocasionó que los aspirantes a líderes tuvieran que buscar conexiones externas entre los caciques de América del Sur. Según esta teoría, esta orientación hacia el sur estuvo acompañada por pequeñas migraciones provenientes de América del Sur hacia Centroamérica y por la sustitución de jade por oro como bien de prestigio. No obstante, la reciente comparación de cinco trayectorias de cambio social para el territorio costarricense (Murillo Herrera 2010) ha concluido que no existe evidencia alguna de que la activación del intercambio a larga distancia, ya sea con Mesoamérica o con América del Sur, haya estado acompañado de transformaciones sociopolíticas en las sociedades precolombinas de lo que hoy es Costa Rica.

Además, resulta altamente conspicuo que en el sector del Pacífico Norte de Costa Rica no se haya detectado una jerarquía de asentamientos en ningún momento de su secuencia, a pesar de ser la región que ha sido utilizada como referente en cuanto a intercambios a larga distancia, en este caso con Mesoamérica. Lo mismo ocurre con la Bahía Neguanje en la costa Caribe colombiana, donde 
se ha relacionado material cultural encontrado con contactos con Centroamérica (Bischof 1969, aunque contrastar con Sáenz Samper \& Lleras Pérez 1999). Investigaciones recientes (Langebaek 2005: 118) no han encontrado evidencia alguna que apoye un escenario de surgimiento de jerarquías políticas regionales.

En resumen, si bien se ha especulado mucho sobre el impacto que pudieron tener contactos e interacciones entre sociedades lejanas ubicadas en Mesoamérica, el istmo Centroamericano y el norte de Colombia, la evidencia por ahora disponible indica que dichos contactos no tuvieron un impacto directo, decisivo o importante sobre el desarrollo social de los grupos locales, lo cual apoya observaciones hechas al respecto años atrás (e.g. Fitzgerald Bernal 1996; Linares Tribaldos 1979; Sheets 1992).

Por otra parte, desde hace al menos veinte años, la evidencia arqueológica se ha intentado relacionar con la información tanto genética como la lingüística, para argumentar a favor de un desarrollo ideológico, político y económico común entre las poblaciones autóctonas de estos territorios. Dicha evidencia apunta a que, las poblaciones aborígenes, en la región que fue mencionada, compartían una base genética (Barrantes et al. 1990; Barrantes 1993, Melton et al. 2013) y lingüística común (Constenla Umaña 1991, 1995; Quesada Pacheco 2007), la cual existió antes del 4000 a.C. Es de esta manera que se han postulado conceptos tales como "Región Histórica Chibcha", "Área de Tradición Chibchoide" (Fonseca Zamora 1992, 1994, 1997a, 1997b.), "Región Histórica Chibcha-Chocó” (Cooke 1992; Fonseca Zamora \& Cooke 1994), "Área Istmo-Colombiana" (Hoopes \& Fonseca Zamora 2003) y "el Mundo Chibcha” (Hoopes 2005) para el territorio comprendido entre el sureste de Honduras y el noroeste de Colombia. Estos conceptos no solo se han utilizado para simplemente definir un área geográfica, sino también para describir un mismo "fenómeno sincrónico y diacrónico... un movimiento social, una dinámica social" (Fonseca Zamora 1997a, 324) el cual ha sido definido como una "unidad difusa" de "no solo tradiciones técnicas, sino también de un patrimonio ideacional [sic]...el cual condicionó la estructuración [sic] del poder entre la gente que hablaba Chibcha, desde los primeros siglos antes de Cristo hasta el siglo dieciséis" (Hoopes \& Fonseca Zamora 2003: 52, traducción nuestra). Es así como los autores plantean el Área IstmoColombiana ${ }^{3}$ no solo como una definición de un espacio geográfico, sino como un mismo proceso "holístico" (Hoopes \& Fonseca 2003: 63) de desarrollo social precolombino en Costa Rica, Panamá y Colombia. Para estos autores, "algunos 'amplios fenómenos horizontales' y algunas 'tradiciones culturales unificadoras' pudieron haber desempeñado una función directa en el surgimiento de la complejidad social, tal como se define por la aparición de la desigualdad social, la jerarquía y la formalización de instituciones religiosas, políticas y económicas" (Hoopes 2005: 5 , traducción nuestra). Por lo tanto, claramente, estamos frente a un modelo que pretende explicar, o al menos dar cuenta del cambio social en la región, a partir del estudio de horizontes y tradiciones culturales (Hoopes 2004, 2005, 2011; Hoopes \& Fonseca 2003).

Pero, de acuerdo con los autores, ¿cómo se podría llegar a conocer si la explicación que ha sido ofrecida es correcta? Examinando "fenómenos horizontales amplios" y "tradiciones culturales unificantes" para ver si estos "pudieron haber desempeñado un papel directo en el surgimiento de la complejidad social" (Hoopes 2005: 5, traducción nuestra), pero al mismo tiempo, estos elementos son, también, producto de "una sucesión compartida de cambios culturales endógenos y realidades similares" (Hoopes \& Fonseca 2003: 53), entonces no se podría justificar el modelo a través del estudio de horizontes y tradiciones arqueológicas, dado que estaríamos entrando en circularidad argumentativa (la causa no puede ser, al mismo tiempo, la consecuencia). Además, desde ya hace mucho tiempo en la práctica de la arqueología (e.g. Binford 1962; Flannery 1967; Taylor, 1948) se ha señalado que no existe una relación directa entre una descripción estilística y artefactual

3 En el resto de la discusión se utilizará este nombre dado, que ha sido el nombre más reciente que le dieron sus dos principales proponentes. 
y la comprensión de las características sociales de los grupos humanos que los produjeron. Entonces, se tendría que recurrir a evidencia externa a las tradiciones y a los horizontes arqueológicos, dado que éstas se proponen, también, como la causa para conocer si estos tuvieron un peso relevante sobre los procesos de transformación social o no. No obstante, se tendría que recuperar evidencia concerniente al fenómeno que se desea conocer en último caso, es decir, al "surgimiento de la complejidad social, tal como se define por la aparición de la desigualdad social, la jerarquía y la formalización de instituciones religiosas, políticas y económicas" (Hoopes 2005: 5, traducción nuestra). La recopilación de información arqueológica acerca de variables tales como tamaño de población (esto está, estrechamente, relacionado con el cambio social de diversas maneras), distribución espacial de asentamientos (lo cual está relacionado, directamente, con el uso de recursos, la centralización político-social, la guerra y otros factores relevantes), distribución espacial de los diversos tipos de artefactos (lo cual está relacionado, fuertemente, con patrones de rango social, especialización artesanal, contactos con otras regiones y otros factores relevantes) que vendrían a ser, entonces, muy útiles y relevantes. $\mathrm{Si}$ se reconstruyen las secuencias de cambio de estas variables sociales y si se contrastan con los horizontes y las tradiciones arqueológicas se podría, entonces, observar cuándo y dónde estas variables coinciden y cuándo y dónde no. En conclusión, si lo que los investigadores desean es conocer si hubo o no una diseminación de ciertos motivos y de ciertas tecnologías entre sociedades; la detección de similitudes y rupturas en los aspectos estilísticos y formales del material arqueológico va a ser muy útil, sin embargo, si la pregunta tiene como objetivo la comprensión de los desarrollos sociales en el pasado de la región, entonces la evidencia arqueológica relevante va a ser definitivamente otra.

Hay otros problemas presentes en el modelo de cambio social que ha sido propuesto en la definición de un "Área Istmo-Colombiana” sobre los cuales es preciso referirse. Por un lado, los estudios lingüísticos y genéticos son claros en señalar que los nodos iniciales de la divergencia de las lenguas chibchas y de su base genética parecen coincidir una sola vez hace varios miles de años a.C., más precisamente antes del 4000 a.C. (Barrantes et al. 1990: 80; Constenla Umaña 1991: 187-189; Fonseca, 1997: 327; Quesada Pacheco 2007: 17-48). No obstante, posterior a esta fecha, una divergencia genética, lingüística y arqueológica temprana entre poblaciones del istmo y de Colombia ha sido documentada, ampliamente y ha sido descrita (Constenla Umaña 1991, 1995; Langebaek 2005: 14-16; Melton et al. 2013; Quesada 2007: $44,235)$, por lo tanto, no parece adecuado extrapolar dicha coincidencia a desarrollos sociales posteriores al 4000 a.C. Por otro lado, siguiendo en la misma línea argumentativa anterior, al utilizar la información proveniente de estudios lingüísticos y genéticos como evidencia a favor e independiente del modelo Istmo-Colombiano, los proponentes del mismo están asumiendo que los procesos de cambio lingüísticos y genéticos están sincronizados entre sí y que estos, a su vez, también lo están con otros procesos sociales que la arqueología estudia, como los tecnológicos, los políticos, los ideológicos y los económicos de allí, el uso del concepto de "unidad difusa" y de enfoques holísticos (e.g. Hoopes \& Fonseca Zamora 2003; Hoopes 2005). Es difícil de imaginar cómo se puede esquivar el hecho de que, usualmente, la evidencia indica exactamente lo opuesto: estos elementos sociales suelen ser independientes debido a los procesos de divergencia social que se gestan a partir de las poblaciones fundacionales, hecho documentado en Europa (Gamble et al. 2005) México (Flannery \& Marcus 1983; Sanders \& Webster 1979), Polinesia (Kirch 1984), Madagascar (Kottak 1977) y Panamá (Linares Tribaldo \& Ranere 1980). Por lo tanto, no se debería esperar que los radios de cambio de las diferentes variables sociales sean coincidentes, ni siquiera aproximadamente. Si la coincidencia existiera, sería entonces la excepción, no la norma, pero primero habría que demostrar que la coincidencia existió posterior al 4000 a.C.

Finalmente, si bien puede existir continuidad geográfica o temporal en formas, estilos y diseños presentes en el material cultural, sus significados 
y contenidos pueden ser totalmente diferentes en tan solo unos pocos años y en tan solo algunas decenas de kilómetros (Panofsky 1939: 3-17; Wobst 1977), sin embargo, no resulta nada convincente, simplemente asumir que ciertas "ideas" son transmitidas junto con ciertos diseños u objetos sin mayor evidencia disponible. Un leve intento por parte de investigadores para evitar este non sequitor ha consistido en usar información etnohistórica, pero este proceder no elimina la distancia temporal y, en muchas ocasiones tampoco la étnica, entre la sociedad precolombina y la sociedad aborigen colonial, ni toda la problemática en torno a los sesgos de los cronistas. La urgencia de "llenar" el vacío entre los artefactos y el mundo de las ideas precolombinas ha llevado a muchos investigadores a hacer uso acrítico y desmedido de la evidencia etnohistórica, esto a pesar de las múltiples advertencias en torno a su uso y abuso (e.g. David \& Kramer 2001; Flannery $\&$ Marcus 1993).

\section{Especialización e intercambio a corta distancia}

En el norte de Colombia, ya desde mediados de la década de 1980 , se venían planteando modelos de desarrollo local que poco a poco se alejaban de explicaciones centradas en influencias e intercambio a larga distancia y se enfocaban cada vez más en dinámicas de intercambio local o de "media distancia” (Angulo Valdés 1988; Cárdenas Arroyo 1988; Langebaek 1987b; OyuelaCaycedo 1986, 1987a, 1987b). Por ejemplo, la existencia de una compleja y extensa red de caminos en la Sierra Nevada de Santa Marta (Serje de la Ossa 1984, 1987) y la diversidad de microambientes presentes en la zona motivó la propuesta de un sistema de "micro-verticalidad" para la producción de bienes de subsistencia (Cárdenas Arroyo 1988, Langebaek 1987b, Oyuela-Caycedo 1990) el cual pudo haber propiciado la interacción y el intercambio entre distintas comunidades en la región. Es interesante, entonces, notar que, dado que no parece haber existido una fuerte integración sociopolítica entre las comunidades precolombinas en ningún momento de la secuencia de la Sierra Nevada (Oyuela-Caycedo 1995), la interacción y el intercambio local no parece haberse vinculado, en este caso, con un proceso de centralización política. Por otra parte, recientemente, la naturaleza del surgimiento de aldeas especializadas y su relación con el surgimiento de complejidad social en la región ha sido estudiada en la Bahía de Chenge (Dever Fonnegra 2007). La evidencia, que ha sido encontrada, sugiere que la especialización comunitaria en la producción de sal y el desarrollo de redes de intercambio parece haber estado vinculada, estrechamente, al surgimiento de jerarquías sociopolíticas, sin embargo, dicha especialización antecedió al surgimiento de líderes locales y más bien tuvo su origen dentro de una relación horizontal entre unidades domésticas.

En el caso de Costa Rica, se ha argumentado que la especialización en la producción agrícola pudo haber estado relacionada con procesos de concentración de la población en aldeas y la complejización de las sociedades ubicadas en la región del Pacífico Sur (Drolet 1988), aunque la evidencia al respecto aún es bastante preliminar. En contraste en el Pacífico Norte la evidencia indica que el intercambio fue esporádico y no a través de redes formalizadas por las élites (Creamer 1992). Investigaciones, que fueron desarrolladas en San Ramón de Alajuela (Murillo Herrera 2010, 2011), señalan que, si bien hubo algún tipo de intercambio entre poblaciones ubicadas en regiones vecinas del Pacífico Central y Norte, el intercambio no fue sincrónico, ocurrió en épocas distintas y, además, no parece haber tenido efecto alguno en la forma en que las sociedades comparadas se desarrollaron sociopolíticamente. Si bien pudieron haber colaborado al estímulo de los eventos de cambio político, de ninguna manera señalaron la dirección de este. Adicionalmente, hubo poca especialización en la región de San Ramón, particularmente a lo interno de sus aldeas y la redundancia en actividades productivas por parte de cada unidad doméstica se resalta aún más si se comparada con otras regiones del norte de América del Sur (Martín \& Murillo Herrera 2014). 
Parece ser que la misma observación es válida para el valle del río La Villa en Parrita, en la Región Central de Panamá (Isaza Aizpurúa 2007). No obstante, un poco más hacia el norte, en el valle del río Parrita el establecimiento de redes de intercambio locales y regionales parece haber estado relacionado con el surgimiento de sociedades cacicales (Haller 2008), situación que también se detectó a escala comunitaria, en el surgimiento de una aldea principal en dicha región (Menzies 2009; Menzies \& Haller 2012a). La eventual competencia por controlar dichas redes de intercambio, por parte de aspirantes a líderes ubicados en distintas aldeas (por ejemplo, El Hatillo y Conte) de la Región Central de Panamá, pudo haber originado la presencia de "jefaturas cíclicas" entre el 700 y el 1522 a.C. (Menzies \& Haller 2012b). El reciente descubrimiento de ricos ajuares funerarios, similares y coetáneos a los encontrados en el sitio Conte (entre el 700 y el 1000 d. C.), en el sitio El Caño (Mayo, J. \& Mayo, C. 2013), refuerzan la tesis de que, para el caso de la Región Central de Panamá, el acceso a las redes de intercambio estaba relacionado con una diferenciación social fuertemente basada en los bienes de prestigio.

\section{Medio ambiente, subsistencia, intensificación agrícola}

Como Archila Montañez (1993: 2) ha señalado, si bien es común entre investigadores el hacer hincapié en la importancia que los factores y los recursos ambientales tuvieron para las poblaciones habitantes y en los cambios en estas, el comprobar o el rechazar esta causalidad requiere de estudios específicos enfocados en este tema y no simplemente resaltar, de manera general, el vínculo obvio entre el ser humano y su entorno medioambiental. Justamente ha sido a través de este tipo de enfoque que se ha logrado determinar que entre el 4000 a.C. y el 1100 d.C. en las tierras bajas del Caribe colombiano "los datos arqueológicos no permiten afirmar que los factores ambientales estuvieran relacionados, directamente, con cambios en las formas de subsistencia o en la distribución de los asentamientos" (Archila Montañez 1993: 15). De igual manera, en la región de Tilarán en Costa Rica, Sheets (1994) ha argumentado que a pesar de las inestabilidades medioambientales que fueron ocasionadas por las erupciones del Volcán Arenal, las sociedades locales tuvieron un modo de vida estable por cerca de 4000 años, manteniendo una organización política simple y una dieta variada y no especializada.

No obstante, en otras regiones del norte de Colombia y en el sur de América Central parece existir un vínculo causal entre medio ambiente y desarrollo social. Así, por ejemplo, al comparar las trayectorias de cambio social presentes en el valle del río Parita y en el valle del río Tonosí en Panamá, Berrey (2014) ha señalado que las diferencias regionales en cuanto a riesgo medioambiental pudieron haber tenido un impacto significativo en el nivel de jerarquización diferenciado que se presentó en ambas regiones. Así mismo, en el río Ranchería en la Guajira colombiana, Langebaek, Cuéllar $\&$ Dever (1998), han encontrado evidencia de que el desarrollo temprano de sociedades aldeanas, alrededor del 1 a.C., y el posterior incremento en la densidad y en el tamaño de la población después del 250 d.C. fue, probablemente, truncado hacia el 1300 d.C., debido a una fuerte sequía que desertificó la zona. Mientras tanto, la presencia de ocupación focalizada, pero no exclusiva en la Bahía de Cinto, en Santa Marta, Colombia, parece haber estado relacionada con suelos más fértiles y condiciones menos secas (Langebaek 2005: 118). Por otra parte, Oyuela-Caycedo (2008) ha propuesto que una crisis catastrófica del medio ambiente en la región de Santa Marta, alrededor del 500 d.C., estimuló el intercambio interregional y la especialización, lo que llevó al surgimiento de sociedades cacicales. No obstante, si bien en las bahías de Santa Marta se detecta un periodo seco alrededor de esa fecha, no se han encontrado evidencias de una crisis demográfica (Langebaek 2005: 118). Mientras que, en Panamá, Isaza Aizpurúa (2007), ha señalado que la riqueza en recursos costeros fue 
vital en el desarrollo de complejidad social en el valle del río La Villa en Parita.

Ciertamente, en el sur de América Central y en Colombia la producción de maíz se continúa considerando como el elemento clave para la producción de excedentes, el incremento sustancial de la población y el surgimiento de la complejidad social en la región (Archila Montañez 2008: 72). Es así como, para el caso de Costa Rica, se ha argumentado que fue la completa adopción del maíz alrededor del 500 a.C. lo que produjo los excedentes de alimento necesarios para el desarrollo de la complejidad social (Corrales Ulloa 2000: 31; Fonseca, 1992; Snarskis 1978, 1984, 1986, 2003). No obstante, este escenario aún no ha sido estudiado en el registro arqueológico. Aún si se lograra acumular evidencia para sostener ese escenario para alguna región del país, no podría generalizarse para otras regiones, teniendo en cuenta que, por ejemplo, en San Ramón de Alajuela el surgimiento de una sociedad cacical ocurrió alrededor del 900 d.C., es decir, al menos 900 años después de que las sociedades en la región desarrollaran una vida aldeana completamente sedentaria y al menos 1900 años después de la completa adopción del maíz en la Región Central de Costa Rica (Arford \& Horn 2004; Lane, Horn \& Mora 2004). Además, como afirma Archila Montañez (2008: 77) "muchos grupos humanos de los trópicos practicaron la producción de alimentos al menos 5000 años antes de que emergiera la vida en aldeas".

$\mathrm{Al}$ sur de las llanuras del Caribe colombiano se ha documentado la presencia de un complejo sistema hidráulico en la depresión Momposina, el cual incluyó la construcción de campos elevados en un área de más de 500.000 hectáreas, desde al menos el 1000 a.C. hasta alrededor del 1300 d.C. (e.g. Berrio et al., 2001; Herrera 2006; Herrera et al. 2001; Herrera, Rojas \& Montejo 2004; Plazas \& Falchetti 1981a, 1981b, 1986, 1990; Plazas \& Falchetti, 1990; Plazas et al. 1988; Plazas et al. 1993; Rojas Mora \& Montejo Gaitán 2006; Rojas, 2008; Rojas, 2010). Los campos elevados fueron utilizados por los habitantes precolombinos para enfrentar las inundaciones periódicas de la región y adecuar tierras para la habitación humana y para la producción e intensificación agrícola (Plazas et al. 1993: 9-18, 126; Herrera 2006; Herrera, Rojas \& Montejo 2004: 148-149) en una superficie para uso potencial de aproximadamente 6.167 ha en el bajo río San Jorge (Rojas, 2010, 301). Si bien en la depresión Momposina es donde estas obras alcanzan la mayor expresión en el Caribe colombiano, este tipo de manejo hidráulico de las zonas inundable parece ser un proceso adaptativo generalizado en distintas regiones de las llanuras de dicha región (Plazas et al. 1993: 11). Respecto a la relación entre la construcción masiva de campos elevados y el desarrollo social precolombino en la región, si bien algunos arqueólogos afirman que éstos estuvieron relacionados al desarrollo de un sistema social complejo y jerarquizado en la región (e.g. Plazas \& Falchetti 1981; Plazas et al. 1993) otros autores (Rojas Mora \& Montejo Gaitán 2006; Rojas, 2008, 2010) han externado, abiertamente, su escepticismo a dicha relación y han señalado que si bien se han avanzado mucho respecto al tema de la reconstrucción medio ambiente en esta región, poco se ha avanzado respecto a variables tales como patrones de asentamiento, cambios demográficos, intercambio de bienes de prestigio, entre otras (Rojas, 2008: 280) y, por lo tanto, es prematuro hablar del desarrollo de sociedades jerarquizadas en esta región. E incluso han propuesto escenarios de desarrollo social no jerarquizados y de alta estabilidad en la región (Rojas Mora \& Montejo Gaitán 2006; Rojas, 2008).

Por otra parte, en el Valle de Aburrá, Langebaek y sus colaboradores no encontraron "una relación clara entre el énfasis en la agricultura y una densidad de población considerable" (Langebaek et al. 2002: 82). Sin embargo, ésta sí pareció existir en el Bajo Magdalena, justo después de establecerse la vida sedentaria en la región, no obstante, no hay mayor indicio de complejización política (Langebaek \& Dever 2000). Relacionado a esto, al otro extremo de la región en estudio, en las tierras altas del Valle del Térraba en el suroeste de Costa Rica, un vínculo entre complejidad política y cambios tecnológicos que están relacionados con actividades agrícolas sí parece haber estado presente (Sol Castillo 2013), dado que variables tales como agregación de personas en aldeas nucleadas y el surgimiento de arquitectura 
monumental parecen haber estado vinculados a cambios en la organización y en la tecnología de la producción agrícola precolombina.

\section{Presión demográfica y guerra}

Si bien se ha argumentado que la presión demográfica fue importante para el surgimiento de sociedades cacicales en Costa Rica (Snarskis 1978: 296, 1984: 230, 1986), en realidad no existe indicio alguno de que existió presión demográfica sobre los recursos en ningún periodo precolombino y en ninguna de las regiones que, hasta hoy, han sido estudiadas. Si se toma en cuenta la evidencia muy indirecta, por ejemplo, no existen datos sobre aumentos abruptos y substanciales de población en las secuencias de Arenal, Gran Nicoya, Pacífico Central y Diquís (Murillo Herrera 2010). Por otra parte, en el caso de San Ramón de Alajuela (Murillo Herrera 2010, 2011) se ha documentado un marcado incremento de la población regional en el periodo anterior al surgimiento de una sociedad cacical, no obstante, el surgimiento de la misma coincide con una leve disminución del tamaño de la población. Mientras que, para el caso de Panamá (Berrey 2014), la divergencia en jerarquización entre las sociedades ubicadas en el valle del río Parita y las que habitaron el valle del río Tonosí parece haber sido provocada, entre otros factores, por niveles diferenciales de crecimiento demográfico regional.

Los resultados del trabajo de Langebaek y asociados en el norte de Colombia han venido a contradecir la idea de que el crecimiento de población fuera determinante en el origen de sociedades complejas que allí surgieron. En el Valle de Aburrá (Langebaek et al. 2002), la evidencia de crecimiento de población surge después del desarrollo de centros regionales. De manera similar, en el Ranchería Medio de la Guajira, la población aumentó, significativamente, solamente después del aparente surgimiento de la complejidad social en la región (Langebaek, Cuéllar \& Dever 1998: 53). En la región de Santa Marta cuando los investigadores detectan una alta densidad de población en las bahías "es evidente que se está haciendo referencia a un fenómeno posterior a las primeras evidencias de complejidad social que les preceden" (Langebaek 2005: 90). Ante lo cual Langebaek ha propuesto que es poco probable que la presión demográfica en esa zona del litoral pudiera relacionarse con el proceso de ocupación de la Sierra Nevada. Por otra parte, para el caso del bajo Magdalena, estudios preliminares indican una disminución de la población a partir del 800 a.C. y hasta la llegada de los españoles (Langabek \& Dever 2000: 51-52), aunque los investigadores han indicado que sus datos son aún muy localizados como para extenderlos a toda la región. En resumen, la evidencia hasta ahora obtenida señala que en el norte de Colombia el surgimiento de complejidad social se originó en condiciones de baja densidad de población (Langebaek 2005: 90).

En Costa Rica, la evidencia de guerra sigue siendo aún muy indirecta, dependiendo, principalmente, de información etnohistórica y de las representaciones de guerreros en la estatuaria precolombina. Si bien ha habido algunos tímidos acercamientos a través de la arqueología (Findlow, Snarskis \& Martin 1979: 64), aún no se ha encontrado evidencia alguna de fronteras o posiciones de defensa bien delimitadas para poder hablar de algún nivel de intensidad de conflictos bélicos (Snarskis 1981: 55, 2003: 193). Así, por ejemplo, la ausencia de zonas de amortiguamiento y la presencia de un patrón de asentamiento disperso en las zonas altas del Valle del Térraba en el sureste de Costa Rica sugieren que la guerra no fue un elemento preponderante en la organización social en dicho lugar (Sol Castillo 2013). En el Valle de Aburrá (Langebaek et al. 2002), en Colombia, la evidencia de guerra ligada a aumento de población y al surgimiento de conflicto proviene, únicamente, de la región de Girardota, la cual posee los suelos menos fértiles del Valle, lo cual va claramente en contra de teorías que relacionan estas variables. A pesar de lo conspicuo de los hallazgos en el sitio Conte, las recientes investigaciones regionales llevadas a cabo en la Región Central de Panamá (Haller 2008; Isaza Aizpurúa 2007), no han logrado encontrar evidencia 
arqueológica que indique algún nivel conspicuo de intensidad bélica entre poblaciones precolombinas.

\section{Festividades, rituales y gerencia}

Recientemente, (Giraldo 2010: 290) ha propuesto que el surgimiento de sociedades cacicales en Ciudad Perdida y Pueblito pudo haber estado vinculado a actividades festivas auspiciadas por una élite la cual controlaba y dirigía los trabajos constructivos. De forma similar, en la región del Volcán Barú en Panamá (Palumbo 2009, 2010), el patrocinio de actividades festivas parece haber contribuido al surgimiento de complejidad social, estas actividades pudieron haber sido complementadas por las élites incipientes con el control de la producción artesanal lítica.

En San Ramón de Alajuela, en Costa Rica, parece ser que las actividades especializadas en el ritual y en las festividades no tuvieron lugar en los asentamientos más densamente poblados ni en los de mayor tamaño. La evidencia indica que las actividades de carácter ritual o festivo en la región tuvieron lugar en asentamientos donde se invirtió trabajo en la construcción de una arquitectura diferenciada. Más precisamente, las aldeas más, densamente, pobladas en dicha región no coinciden con las aldeas donde se desarrolló arquitectura de carácter monumental (Murillo Herrera 2011: 60-68). Mientras que los asentamientos con arquitectura monumental y que acapararon actividades de índole festivo o ceremonial se ubicaron en sectores de la región con fácil acceso al ingreso de bienes foráneos (Bergoeing \& Murillo Herrera 2013). No obstante, más relevante para la presente temática resultan las investigaciones recientes en las tierras altas de Valle del Térraba, una zona rica en petrograbados y estatuaria en general. Los datos arqueológicos que fueron analizados, desde una perspectiva regional (Sol Castillo 2013), indican que si bien los ritos de enterramientos cumplieron una función relevante en la constitución y en el mantenimiento de linajes, así como en los procesos de integración social, las actividades religiosas o rituales, éstos no fueron los elementos sociales principales en el surgimiento de la complejidad social en la región.

\section{Acerca de los avances de investigación}

Como se ha mostrado, se han dado avances substanciales en el estudio de los factores que subyacen a los procesos de desarrollo social precolombino. Estos avances indican que la identificación de un solo factor (intercambio) y un solo mecanismo (interacción interregional) para hablar de cambio social precolombino en América Central o América del Sur, no solo resulta apresurado, sino también ficticio, teniendo en cuenta la evidencia disponible. Por otra parte, sería también descuidado creer que, porque no se pueda señalar una sola causa de cambio y porque no se pueda describir sus múltiples manifestaciones y procesos, entonces, el estudio del cambio social deba considerarse como ininteligible o caótico; por el contrario, lo que implica es más bien que queda mucho camino por recorrer antes de poder señalar patrones mayoritariamente predominantes en la región.

Así, por ejemplo, a partir de la información arriba parece indicar que la especialización en la producción de bienes y la activación de redes de intercambio con regiones aledañas fue una estrategia popular entre algunos líderes en el sur de América Central y el norte de Colombia, con el objetivo de incrementar su prestigio social. Ciertamente, hoy en día, existen investigaciones bastante sistemáticas que han aportado información local en esa dirección. Sin bien es cierto que las investigaciones sistemáticas que son enfocadas en el cambio social, apenas se están implementando en la arqueología de América Central y América de Sur y, por lo tanto, se debe tener cautela acerca de afirmaciones categóricas respecto a las causas detrás del cambio social, es necesario que la cautela no paralice el ofrecimiento de conjeturas informadas y su respectiva investigación empírica. Por otra parte, es importante recordar que el proceso de conocer 
implica descartar explicaciones, por lo tanto, resulta interesante cómo ciertas hipótesis que se han propuesto como teorías generales o universales para el desarrollo de la complejidad social (difusión, presión demográfica, intensificación agrícola, intercambio a larga distancia), tienen poco o ningún sustento empírico en varios de los casos que han sido expuestos arriba.

Como se mencionó al inicio de este artículo, la pretensión no ha sido hacer un recuento exhaustivo de la arqueología del sur de América Central y el norte de Colombia, sino exponer los avances más recientes en cuanto al estudio de los factores implicados con el cambio social precolombino. Al hacer un repaso somero por la información disponible para el desarrollo de las poblaciones originarias que se ubicaron es inevitable notar la extraordinaria diversidad en adaptaciones y procesos de desarrollo social a las que éstas recurrieron. Esta observación no es nueva (Drennan 1996), no obstante, la evidencia acumulada en los últimos 15 años respalda aún más la vigencia de esta observación; incluso para épocas tan tempranas como el Formativo (González Fernández 2005, León \& Salgado González 2005).

Es importante notar que acá se ha estado hablando en todo momento de desarrollos sociales y no de estilos, tradiciones u horizontes de materiales o rasgos arqueológicos. Así, se ha hecho referencia a diversidad social, y no a diversidad material. Muchas de las respuestas en torno a la función precisa que pudieron tener los factores que han sido expuestos arriba respecto al cambio social precolombino en cada secuencia, solo se podrán responder si se incorpora otras escalas de análisis (regional, asentamiento o comunidad, unidad doméstica) que se han emprendido recientemente o que se ejecutarán en el futuro.

Respecto al plano teórico, sería deseable que en el futuro otros estudios comparativos evalúen el tema de diversidad/homogeneidad en cuanto a naturaleza y formas sociopolíticas que se desarrollaron en esta región del mundo, a partir del estudio de las variables pertinentes, como por ejemplo, dispersión/ agregación de la población, surgimiento o no de lugares centrales y la concentración o no de instituciones ideológicas, políticas y económicas; así como en cuanto a la estabilidad e inestabilidad de los aparatos políticos. Estos temas ya están siendo investigados en diversas regiones de América Central y de Colombia y, definitivamente, requieren de mucha más atención si se desea avanzar en la comprensión del cambio social.

Murillo Herrera, Mauricio. Beyond pilgrims and gold: Precolombian social development in Costa Rica, Panama and North Colombia. R. Museu Arq. Etn.,31: 56-79, 2018.

Abstract: This study comprehensively introduces the main advances in understanding the development of the pre-Columbian societies that inhabited Costa Rica, Panama and northern Colombia and a critical assessment of the results is also provided. The main purpose is to increase our understanding of the pre-Columbian social change processes in the region, mainly those that have taken place in the last two decades. The analysis is carried out both for early social developments and for late complex societies, and the latter are studied based on the themes proposed by researchers as the main drivers of social change. The article presents the extraordinary diversity in adaptations and social development processes that occurred in the region during the pre-Columbian era, which goes against the idea of social and cultural homogeneity. On the other hand, it is highlighted that, indeed, there have been advances in the study of ancient societies in the region, but progress has fallen short, because: 1) conceptual elaboration has been confused with an increase in the understanding 
Más allá de peregrinos y de oro: desarrollo social precolombino Costa Rica, Panamá y el norte de Colombia R. Museu Arq. Etn., 31: 56-79, 2018.

of social and economic developments; and 2) it has not been possible to overcome the epistemological and methodological gap between describing the archaeological material and context, and studying social processes in the past. Finally, it is shown that to understand the whole range of pre-Columbian social developments that have been described in these three countries, it is necessary to consider other change factors within the models that have been historically proposed for the region: exchange and interregional interaction.

Keywords: Archaeology; Social change; Colombia; Panama; Costa Rica.

\section{Referencias bibliográficas}

Aceituno Bocanegra, F.J.; Rojas Mora, S. 2012. Del paleoindio al formativo: 10.000 años para la historia de la tecnología lítica en Colombia. Boletín de Antropología 26(43): 124-156.

Angulo Valdés, C. 1978. Arqueología de la Ciénaga Grande de Santa Marta. Fundación de Investigaciones Arqueológicas Nacionales, Bogotá.

Angulo Valdés, C. 1983. Arqueología del Valle de Santiago, norte de Colombia. Fundación de Investigaciones Arqueológicas Nacionales, Bogotá.

Angulo Valdés, C. 1987. Arqueología de la llanura atlántica de Colombia: balances y proyecciones. En: Vargas Arenas, I. (Ed.). Revisión crítica de la arqueología del Caribe. Fundación Arqueología del Caribe, Washington, 37-56.

Angulo Valdés, C. 1988. Relaciones de intercambio entre tres regiones arqueológicas del norte de Colombia (Ciénaga Grande, Sierra Nevada, Bajo Magdalena). En: Fonseca Zamora, O. (Ed.). Hacia una arqueología social. Editorial Universidad de Costa Rica, San José, 78-89.

Angulo Valdés, C. 1995. Modos de vida en la prehistoria de la llanura atlántica de Colombia. Centro de Estudios Regionales, Barranquilla.

Archila Montañez, S. 1993. Medio ambiente y arqueología de las tierras bajas del Caribe colombiano. Boletín Museo del Oro 34-35: 111-164.
Archila Montañez, S. 2008. Modelos teóricos y arqueobotánica en el noroeste de Suramérica. In: Archila, S.; Giovannetti, M.; Lema, V. (Eds.). Arqueobotánica y teoría arqueológica: discusiones desde Suramérica. Ediciones Uniandes, Bogotá, 66-95.

Arford, M.R.; Horn, S.P. 2004. Pollen evidence of the earliest maize agriculture in Costa Rica. Journal of Latin American Geography 3(1): 108-115.

Baldi, N.F.S. 2001. Black Creek (UCR 467): primeras interpretaciones arqueológicas de un modo de vida costero en el Caribe sur de Costa Rica. Tese de Licenciatura. Universidad de Costa Rica, San José.

Baldi, N.F.S. 2011. Explotación temprana de recursos costeros en el sitio Black Creek (4000-2500 A.P.), Caribe sur de Costa Rica. Revista de Arqueología Americana (29): 85-121.

Barrantes, R. 1993. Evolución en el Trópico: los amerindios de Costa Rica y Panamá. Editorial Universidad de Costa Rica, San José.

Barrantes, R. et al. 1990. Microevolution in lower Central America: genetic characterization of the chibcha-speaking groups of Costa Rica and Panama, and a consensus taxonomy based on genetic and linguistic affinity. American Journal of Human Genetics 46(1): 63-84.

Bergoeing, J.P.; Murillo Herrera, M. 2013. El asentamiento precolombino en San Ramón y su 
imbricación geomorfológica. Revista Geográfica 151: 113-127.

Berrey, C.A. 2014. Organization and growth among early complex societies in Central Pacific Panama. Tesis de doctorado. University of Pittsburgh, Pittsburgh.

Berrio, J.C. et al. 2001. Multi-disciplinary evidence of the Holocene history of a cultivated floodplain area in the wetlands of northern Colombia. Vegetation History and Archaeobotany 10(3): 161-174.

Binford, L.R. 1962. Archaeology as Anthropology. American Antiquity 28(2): 217-225.

Bischof, H. 1969. La cultura tairona en el Área Intermedia. In: International Congress of Americanists. Verhandlungen des 38 Internationalen Amerikanistenkongresses, Stuttgart-Munchen, 12. Bis 18, August 1968, Bd. 1. Renner, München, 271-280.

Bradley, J.E. 1994. Tronadora Vieja: an archaic and early formative site in the Arenal Region. In: Sheets, P.D.; McKee, B.R. (Eds.). Archaeology, volcanism, and remote sensing in the Arenal Region, Costa Rica. University of Texas Press, Austin, 73-86.

Bray, W. 1978. Gold-working in Ancient America. Gold Bulletin 11(4): 136-143.

Bray, W. 1984. Across the Darien Gap: a Colombian view of isthmian archaeology. In: Lange, F.W.; Stone, D.Z. The archaeology of Lower Central America. University of New Mexico Press, Albuquerque, 305-338.

Bray, W. 1992. Sitio Conte in its Pan-American context. In: Hearne, P.; Sharer, R.J. (Eds.). River of gold: Precolumbian treasures from Sitio Conte. UPenn Museum of Archaeology and Anthropology, Pennsylvania, 32-46.

Bray, W. 1997. Metallurgy and anthropology: two studies from prehispanic America. Boletín Museo del Oro (42): 38-55.
Bray, W. 2003. Gold, stone, and ideology: symbols of power in the Tairona tradition of northern Colombia. In: Quilter, J.; Hoopes, J.W. Gold and power in ancient Costa Rica, Panama, and Colombia. Dumbarton Oaks Research Library and Collection, Washington, 301-344.

Cadavid Camargo, G.; Groot de Mahecha, A.M. 1987. Buritaca 200: arqueología y conservación de una población precolombina (Sierra Nevada de Santa Marta - Colombia). Beiträge zur Allgemeinen und Vergleichenden Archäologie 4: 255-285.

Callaghan, R.T.; Bray, W. 2007. Simulating prehistoric sea contacts between Costa Rica and Colombia. The Journal of Island and Coastal Archaeology 2(1): 4-23.

Cárdenas Arroyo, F. 1988. Importancia del intercambio regional en la economía del área tairona. Revista Colombiana de Antropología 4(1): 39-64.

Chávez Montoya, M.L.; Naranjo Masís, D. 2013. Gestión integrada del patrimonio arqueológico: experiencias en investigación, conservación y divulgación en el PHR, en Siquirres, Costa Rica. En: Aguilar Bonilla, M.; Niglio, O. (Eds.). La conservación del patrimonio cultural en Costa Rica. Aracne, Roma, 281-304.

Chávez, S.; Fonseca, O.M.; Baldi, N. 1997. Investigaciones arqueológicas en la costa Caribe de Costa Rica, América Central. Revista de Arqueología Americana 10: 123-161.

Constenla Umaña, G. 1991. Las lenguas del Área Intermedia: una introducción a su estudio areal. Editorial Universidad de Costa Rica, San José.

Constenla Umaña, G. 1995. Sobre el estudio diacrónico de las lenguas chibchenses y su contribución al estudio del pasado de sus hablantes. Boletín Museo del Oro (38-39): 13-56.

Cooke, R.G. 1992. Etapas tempranas de la producción de alimentos vegetales en la Baja Centroamérica y partes de Colombia (Región Histórica Chibcha-Chocó). Revista de Arqueología Americana (6): 35-70. 
Más allá de peregrinos y de oro: desarrollo social precolombino Costa Rica, Panamá y el norte de Colombia

R. Museu Arq. Etn., 31: 56-79, 2018.

Cooke, R.G. 1995. Monagrillo, Panama’s first pottery (3800-1200 cal bc): summary of research (19481993), with new interpretations of chronology, subsistence and cultural geography. In: Barnett, W.K; Hoopes, J.W. (Eds.). The emergence of pottery: technology and innovation in ancient societies. Smithsonian Institution Press, Washington, 169-184.

Cooke, R.G. 1998. Cupica (Chocó): A Reassessment of Gerardo Reichel-Dolmatoff's Fieldwork in a Poorly Studied Region of the American Tropics. In: Oyuela-Caycedo, A.; Scott Raymond, J. (Eds.). Recent advances in the archaeology of the northern Andes. Cotsen Institute of Archaelogy, Los Angeles, 91-106.

Cooke, R.G. 2005. Prehistory of native Americans on the Central American land bridge: colonization, dispersal, and divergence. Journal of Archaeological Research 13(2): 129-187.

Cooke, R.G.; Ranere, A.J. 1992. Prehistoric human adaptations to the seasonally dry forests of Panama. World Archaeology 24(1): 114-133.

Cooke, R.G. et al. 2003. Who crafted, exchanged, and displayed gold in pre-Columbian Panama? In: Quilter, J.; Hoopes, J.W. Gold and power in ancient Costa Rica, Panama, and Colombia. Dumbarton Oaks Research Library and Collection, Washington, 91-158.

Corrales Ulloa, F. 2000. An evaluation of long-term cultural change in Southern Central America: the ceramic record of the Diquís archaeological subregion, Costa Rica. Tesis de doctorado. University of Kansas, Lawrence.

Corrales Ulloa, F. 2001. Los primeros costarricenses. Museo Nacional de Costa Rica, San José.

Corrales Ulloa, F. 2006. Excavando Los Sueños, Pacífico Central de Costa Rica. Vínculos 29(1-2): 33-59.

Correal Urrego, G. 1990. Evidencias culturales durante el Pleistoceno y Holoceno de Colombia. Revista de Arqueología Americana 1: 69-89.
Creamer, W. 1992. Regional exchange along the Pacific Coast of Costa Rica during the Late Polychrome Period, A.D. 1200-1550. Journal of Field Archaeology 19(1): 1-16.

David, N.; Kramer, C. 2001. Ethnoarchaeology in action. Cambridge University Press, New York.

Dever Fonnegra, A. 2007. Social and economic development of a specialized community in Chengue, Parque Tairona, Colombia. Tesis de doctorado. University of Pittsburgh, Pittsburgh.

Dickau, R. 2010. Microbotanical and macrobotanical evidence of plant use and the transition to agriculture in Panama. In: VanDerwarker, A.; Peres, T.M. (Eds.). Integrating zooarchaeology and paleoethnobotany: a consideration of issues, methods, and cases. Springer, New York, 99-134.

Drennan, R.D. 1995. Chiefdoms in northern South America. Journal of World Prehistory 9(3): 301-340.

Drennan, R.D. 1996. Betwixt and between in the Intermediate Area. Journal of Archaeological Research 4(2): 95-132.

Drolet, R.P. 1988. The emergence and intensification of complex societies in Pacific Southern Costa Rica. In: Lange, F.W. (Ed.). Costa Rican art and archaeology: essays in honor of Frederick R. Mayer. University of Colorado Press, Niwot, 163-188.

Falchetti, A.M. 1979. Colgantes "Darién": relaciones entre áreas orfebres del occidente colombiano y centroamericano. Boletín Museo del Oro (4): 1-55.

Falchetti, A.M. 1987. Desarrollo de la orfebrería Tairona en la provincia metalúrgica del norte colombiano. Boletín Museo del Oro (19): 3-24.

Falchetti, A.M. 1993. La tierra del oro y el cobre: parentesco e intercambio entre comunidades orfebres del norte de Colombia y áreas relacionadas. Boletín Museo del Oro (34-35): 3-76.

Findlow, F.J.; Snarskis, M.J.; Martin, P. 1979. Un análisis de zonas de explotación relacionadas 
con algunos sitios prehistóricos de la Vertiente Atlántica de Costa Rica. Vínculos 5(2): 53-71.

Fitzgerald Bernal, C. 1996. Prestige goods in the archaeological sequences of Costa Rican and Panamanian chiefdoms. In: Langebaek, C.H.; Cárdenas Arroyo, F. (Eds.). Caciques, intercambio y poder: interacción regional en el área intermedia de las Américas. Universidad de los Andes, Bogotá, 47-62.

Flannery, K.V. 1967. Culture history vs. cultural process: a debate in American archaeology. Scientific American 217(2): 119-122.

Flannery, K.V.; Marcus, J. (Eds.). 1983. The cloud people: divergent evolution of the Zapotec and Mixtec civilizations. Academic Press, New York.

Flannery, K.V.; Marcus, J. 1993. What is cognitive archaeology? Cambridge Archaeological Journal 3(2): 247-270.

Fonseca Zamora, O.M. 1981. Guayabo de Turrialba and its significance. In: Elizabeth, P.B. (Ed.). Between continents/between seas: precolumbian art of Costa Rica. Harry N. Abrams, New York, 104-111.

Fonseca Zamora, O.M. 1992. Historia antigua de Costa Rica: surgimiento y caracterización de la primera civilización costarricense. Editorial Universidad de Costa Rica, San José.

Fonseca Zamora, O.M. 1994. El concepto de área de tradición chibchoide y su pertinencia para entender Gran Nicoya. Vínculos 18-19(1-2): 209-228.

Fonseca Zamora, O.M. 1997a. La conformación de los espacios históricos, el caso de América Central y el noroccidente colombiano. In: Fonseca Zamora, O.M. Memorias del III Simposio Panamericano de Historia: del 25 al 29 de septiembre de 1995. Instituto Panamericano de Geografía e Historia, Ciudad de México, 321-357.

Fonseca Zamora, O.M. 1997b. La cerámica temprana de Costa Rica en el contexto del área histórica chibchoide (4000-2500 a.P.). Revista de Arqueología Americana (13): 41-68.
Fonseca Zamora, O.M.; Cooke, R.G. 1994. El sur de América Central: una contribución al entendimiento de la región histórica ChibchaChocó. In: Carmack, R.M. Historia antigua de Centroamérica. Facultad Latinoamericana de Ciencias Sociales, San José, 217-276.

Fonseca Zamora, O.M.; Richardson, J.B. 1978. South American and Maya cultural contacts at the Las Huacas site, Costa Rica. vol. 47: Annals of the Carnegie Museum. Carnegie Museum of Natural History, Pittsburgh.

Gamble, C. et al. 2005. The archaeological and genetic foundations of the European population during the Late Glacial: Implications for 'agricultural thinking. Cambridge Archaeological Journal 15(2): 193-223.

Giraldo Peláez, S. 2010. Lords of the Snowy Ranges: politics, place, and landscape transformation in two Tairona Towns in the Sierra Nevada de Santa Marta, Colombia. Tesis de doctorado. University of Chicago, Chicago.

González Fernández, V. 2005. Las culturas arqueológicas de Colombia. In: Organismo Autónomo de Museos y Centros. Iconología y simbolismo en Colombia prehispánica, 18-34. Instituto Colombiano de Antropología e Historia, Bogotá.

Griggs, J.C. 2005. The archaeology of Central Caribbean Panama. Tesis de doctorado. University of Texas, Austin.

Groot de Mahecha, A.M. 1980. Buritaca-200: una fecha de radiocarbono asociada con objetos de orfebrería Tairona. Boletín Museo del Oro 8: 21-34.

Groot de Mahecha, A.M. 1989. La Costa Atlántica. In: Contreras, A.B. et al. Colombia prehispánica: regiones arqueológicas. Instituto Colombiano de Antropología, Bogotá, 19-52.

Haller, M.J. 2008. Asiento Viejo and the development of the Río Parita chiefdom, Panama. University of Pittsburgh Memoirs in Latin American Archaeology Publications, Pittsburgh. 
Más allá de peregrinos y de oro: desarrollo social precolombino Costa Rica, Panamá y el norte de Colombia

R. Museu Arq. Etn., 31: 56-79, 2018.

Hansell, P. 1987. The formative in Central Pacific Panama: La Mula-Sarigua. In: Drennan, R.D.; Uribe, C.A. (Eds.). Chiefdoms in the Americas. University Press of America, Lanham, 119-138.

Helms, M.W. 1979. Ancient Panama: chiefs in search of power. University of Texas Press, Austin.

Herrera, L.F. 2006. Paleoecología en la depresión Momposina 21.000 años de cambios ambientales. In: Valdez, F (Ed.). Agricultura ancestral camellones y albarradas: contexto social, usos y retos del presente. Editorial Abya Yala, Quito, 227-240.

Herrera, L.F. et al. 2001. Evolución ambiental de la depresión Momposina (Colombia) desde el Pleistoceno Tardío a los paisajes actuales. Geología Colombiana 26: 95-121.

Herrera, L.F.; Rojas, S.; Montejo, F. 2004. Poblamiento prehispánico de la depresión Momposina: un sistema integrado de manejo sostenible de los ecosistemas inundables. In: Restrepo Arcila, R.A. Saberes de vida: por el bienestar de las nuevas generaciones. Siglo Del Hombre Editores, Bogotá, 148-173.

Hoopes, J.W. 2004. Atravesando fronteras y explorando la iconografía sagrada de los antiguos Chibchas en Centroamérica meridional y Colombia septentrional. Arqueología del Área Intermedia 6: 129-166.

Hoopes, J.W. 2005. The emergence of social complexity in the Chibchan world of Southern Central America and Northern Colombia, AD 300-600. Journal of Archaeological Research 13(1): 1-47.

Hoopes, J.W. 2011. Culturas chibchas del litoral caribeño: exploración de las conexiones precolombinas entre Colombia y Costa Rica. In: González Fernández, V. (Ed.). Arqueología en el Área Intermedia. Instituto Colombiano de Antropología e Historia, Bogotá, 367-412.

Hoopes, J.W.; Fonseca Zamora, O.M. 2003. Goldwork and Chibchan identity: endogenous change and diffuse unity in the Isthmo-Colombian area. In: Quilter, J.; Hoopes, J.W. Gold and power in ancient
Costa Rica, Panama, and Colombia. Dumbarton Oaks Research Library and Collection, Washington, 48-89.

Isaza Aizpurúa, I.I. 2007. The ancestors of Parita: pre-Columbian settlement patterns in the lower $L a$ Villa Valley, Azuero Peninsula, Panama. Tesis de doctorado. Boston University, Boston.

Kidder, A. 1940. South American penetrations in Middle America. In: Hay, C.L. et al (Eds.). The Maya and their neighbors. D. Appleton-Century Company, New York, 441-459.

Kintigh, K.W. et al. 2014 Grand challenges for archaeology. Proceedings of the National Academy of Science 111 (3): 879-880.

Kirch, P.V. 1984 The evolution of the Polynesian chiefdoms. Cambridge University Press, Cambridge.

Kottak C.P. 1977. The process of state formation in Madagascar. American Ethnologist 4(1): 136-155.

Lane, C.S.; Horn, S.P.; Mora, C.I. 2004. Stable carbon isotope ratios in lake and swamp sediments as a proxy for prehistoric forest clearance and crop cultivation in the Neotropics. Journal of Paleolimnology 32(4): 375-381.

Langebaek, C.H. 1987a. La cronología de la región arqueológica Tairona vista desde Papare, municipio de Ciénaga. Boletín de Arqueología 2(1): 83-101.

Langebaek, C.H. 1987b. Relaciones de los desarrollos del Área Tairona y el intercambio. Boletín de Arqueología 2(2): 32-41.

Langebaek, C.H. 2005. The prehispanic population of the Santa Marta bays: a contribution to the study of the development of the northern Colombia Tairona chiefdoms. University of Pittsburgh Memoirs in Latin American Archaeology Publications, Pittsburgh.

Langebaek, C.H.; Cárdenas Arroyo, F. 1996. Caciques, intercambio y poder: interacción regional en el área 
intermedia de las Américas. Universidad de los Andes, Bogotá.

Langebaek, C.H., Cuéllar, A.; Dever, A. 1998. Medio ambiente y poblamiento en la Guajira: investigaciones arqueológicas en el Ranchería Medio. Universidad de los Andes, Bogotá.

Langebaek, C.H.; Dever, A. 2000. Arqueología en el Bajo Magdalena: un estudio de los primeros agricultores del Caribe colombiano. Universidad de los Andes, Bogotá.

Langebaek, C.H. et al. 2002. Arqueología y guerra en el valle de Aburrá: estudio de cambios sociales en una región del noroccidente de Colombia. Ediciones Uniandes, Bogotá.

León, M.; Salgado González, S. 2005. Los desarrollos sociales de la fase Pavas (300 a.C.-300 d.C.) en el sector occidental del Valle Central. Vínculos 27: 1-18.

Linares Tribaldos, O.F. 1979. What is Lower Central American Archaeology? Annual Review of Anthropology 8: 21-43.

Linares Tribaldos, O.F; Ranere, A.J. (Eds.). 1980. Adaptive Radiations in Prehistoric Panama. Harvard University Press, Cambridge.

Llanos Vargas, H. 1993. Presencia de la cultura de San Agustín en la depresión cálida del Valle del Río Magdalena (Garzón-Huila). Boletín de Arqueología 4(2): 51-66.

Lleras Pérez, R. 1987. La utilización de las áreas libres en Ciudad Perdida. Boletín Museo del Oro 19: 97-116.

Lothrop, S.K. 1940. South America as seen from Middle America. In: Hay, C.L. et al (Eds.). The Maya and their neighbors. D. Appleton-Century Company, New York, 417-459.

Mayo, J.; Mayo, C. 2013. El descubrimiento de un cementerio de élite en El Caño: indicios de un patrón funerario en el valle de río Grande, Coclé, Panamá. Arqueología Iberoamericana 20: 3-27.
Martín, A.J.; Murillo Herrera, M. 2014. Networks of interaction and functional interdependence in societies across the Intermediate Area. Journal of Anthropological Archaeology 36: 60-71.

Mason, A. 1931. Archaeology of Santa Marta, Colombia: the Tairona culture. Part 1: report on field work. Field Museum of Natural History, Chicago.

Menzies, A.C.J. 2009. Craft Specialization and the emergence of the chiefly central place community of He-4 (El Hatillo), Central Panama. Tesis de doctorado. University of Pittsburgh, Pittsburgh.

Menzies, A.C.J.; Haller, M.J. 2012a. Embedded craft production at the late pre-Columbian (A.D. 900-1522) community of He-4 (El Hatillo), central region of Panama. Canadian Journal of Archaeology 36(1): 108-140.

Menzies, A.C.J.; Haller, M.J. 2012b. A macroregional perspective on chiefly cycling in the central region of Panama during the Late Ceramic II period (A.D. 700-1522). Latin American Antiquity 23(4): 449-466.

Melton, P.E. et al. 2013. Microevolution, migration, and the population structure of five Amerindian populations from Nicaragua and Costa Rica. American Journal of Human Biology 25(4): 480-490.

Murillo Herrera, M. 2008. Estado actual y perspectivas de la investigación arqueológica en territorio costarricense. In: Jaramillo, L.G. Arqueología en Latinoamérica: historias, formación académica y perspectivas temáticas. Universidad de los Andes, Bogotá, 41-84.

Murillo Herrera, M. 2010. Diversidad sociopolítica en Costa Rica precolombina. Implicaciones para la comprensión del cambio social. International Journal of South American Archaeology (6): 16-34.

Murillo Herrera, M. 2011. Precolumbian social change in San Ramón de Alajuela, Costa Rica. University of Pittsburgh Memoirs in Latin American Archaeology Publications, Pittsburgh. 
Más allá de peregrinos y de oro: desarrollo social precolombino Costa Rica, Panamá y el norte de Colombia

R. Museu Arq. Etn., 31: 56-79, 2018.

Oyuela-Caycedo, A. 1986. De los Taironas a los Kogi: una interpretación del cambio cultural. Boletín Museo del Oro (17): 32-43.

Oyuela-Caycedo, A. 1987a. Aspectos culturales de las secuencias locales y regionales en los Tairona. In: Drennan, R.D.; Uribe, C.A (Eds.). Chiefdoms in the Americas. University Press of America, Lanham, 213-228.

Oyuela-Caycedo, A. 1987b. Gaira: introducción a la ecología y arqueología del litoral de la Sierra Nevada de Santa Marta. Boletín Museo del Oro (19): 35-55.

Oyuela-Caycedo, A. 1987c. Dos sitios arqueológicos con desgrasante de fibra vegetal en la serranía de San Jacinto (departamento de Bolívar). Boletín de Arqueología 2: 5-21.

Oyuela-Caycedo, A. 1990. Las redes de caminos prehispánicos en la Sierra Nevada de Santa Marta. In: Camargo, S.M. Ingenierías prehispánicas. Fondo FEN, Bogotá, 47-71.

Oyuela-Caycedo, A. 1993. Sedentism, food production and pottery origins in the tropics: the case of San Jacinto 1, Colombia. Tesis de doctorado. University of Pittsburgh, Pittsburgh.

Oyuela-Caycedo, A. 1995. Centralización e integración en la Sierra Nevada de Santa Marta. Boletín Museo del Oro (38-39): 113-133.

Oyuela-Caycedo, A. 1996. The study of collector variability in the transition to sedentary food producers in northern Colombia. Journal of World Prehistory 10(1): 49-93.

Oyuela-Caycedo, A. 2008. Late pre-Hispanic chiefdoms of northern Colombia and the formation of anthropogenic landscapes. In: Silverman, H.; Isbell, W. Handbook of South American archaeology. Springer, New York, 405-428.

Oyuela-Caycedo, A.; Bonzani, R.M. 2005. San Jacinto 1: a historical ecological approach to an archaic site in Colombia. University of Alabama Press, Tuscaloosa.
Palumbo, S.D. 2009. The development of complex society in the Volcán Barú region of western Panama. Tesis de doctorado. University of Pittsburgh, Pittsburgh.

Palumbo, S.D. 2010. Rock art and domestic organization at Sitio Barriles, Chiriquí province, Panama. Revista de Arqueología Americana 28: 151-170.

Panofsky, E. 1939. Studies in iconology: humanistic themes in the art of the Renaissance. Oxford University Press, Oxford.

Piperno, D.R.; Bush, M.B.; Colinvaux, P.A. 1991. Paleoecological perspectives on human adaptation in Panama. I. The Pleistocene. Geoarchaeology 6(3): 201-226.

Piperno, D.R. et al. 2000. Starch grains reveal early root crop horticulture in the Panamanian tropical forest. Nature 407(6806): 894-897.

Plazas, C.; Falchetti, A.M. 1981a. Asentamientos prehispánicos en el bajo río San Jorge. Fundación de Investigaciones Arqueológicas Nacionales, Bogotá.

Plazas, C.; Falchetti de Sáenz, A.M. 1981b. El legendario Zenú. Boletín Museo del Oro 4: 20-33.

Plazas, C.; Falchetti, A.M. 1986. La cultura del oro y el agua. Un proyecto de reconstrucción. Boletín Cultural y Bibliográfico 23(6): 57-72.

Plazas, C.; Falchetti, A.M. 1990. Una cultura anfibia: la sociedad hidráulica Zenú. In: Jimeno, M.C. Caribe Colombia. 191-202. Fondo FEN Colombia, Bogotá.

Plazas, C. et al. 1988. Cambios ambientales y desarrollo cultural en el bajo río San Jorge. Boletín Museo del Oro 20: 54-88.

Plazas, C. et al. 1993. La sociedad hidráulica Zenú: estudio arqueológico de 2.000 años de historia en las llanuras del Caribe colombiano. Banco de la República, Bogotá.

Quesada Pacheco, J.D. 2007. The Chibchan languages. Editorial Tecnológica de Costa Rica, Cartago. 
Quilter, J.; Hoopes, J.W. (Eds.) 2003. Gold and power in ancient Costa Rica, Panama, and Colombia. Dumbarton Oaks Research Library and Collection, Washington.

Ranere, A.J.; Cooke, R.G. 1996. Stone tools and cultural boundaries in prehistoric Panama: an initial assessment. In: Lange, F.W. Paths to Central American prehistory, 49-77. University Press of Colorado, Niwot.

Ranere, A.J; Cooke, R.G. 2003. Late glacial and early Holocene occupation of Central American tropical forests. In: Mercarder, J. (Ed.). Under the canopy. The archaeology of tropical rain forests. Rutgers University Press, New Brunswick, 219-248.

Ranere, A.J.; Hansell, P. 1978. Early subsistence patterns along the Pacific Coast of Panama. In: Stark, B.L.; Voorhies, B. Prehistoric coastal adaptations. Academic Press, New York, 43-59.

Ranere, A.J.; López Castaño, C.E. 2007. Cultural diversity in late Pleistocene/early Holocene populations in Northwest South America and Lower Central America. International Journal of South American Archaeology (1): 25-31.

Reichel-Dolmatoff, G. 1965. Colombia, ancient peoples and places. Thames \& Hudson, London.

Reichel-Dolmatoff, G. 1983. Colombia indígena, período prehispánico. In: Manual de historia de Colombia. vol. 1. Círculo de Lectores, Bogotá, 33-224.

Reichel-Dolmatoff, G. 1989. Colombia indígena, periodo prehispánico. In: Uribe, J.J. et al. Nueva historia de Colombia. vol. 1. Planeta, Bogotá, 27-61.

Reichel-Dolmatoff, G. 1990. La Sierra Nevada: cambio cultural y conciencia ambiental. In: Jimeno, M.C. Caribe Colombia. 191-202. Fondo FEN Colombia, Bogotá, 203-224.

Reichel-Dolmatoff, G. 1997. Arqueología de Colombia: un texto introductorio. Biblioteca Familiar Presidencia de la República, Bogotá.Reyes
Paniagua, E.J. 2009a. Unidad y heterogeneidad durante el periodo Formativo en Costa Rica (2000-300 a.C.), una propuesta de interacción cultural. Cuadernos de Antropología (19): 57-74.

Reyes Paniagua, E.J. 2009b. Relaciones culturales en Costa Rica y a nivel regional durante el periodo formativo: movilidad más allá de la región istmo-colombiana. International Journal of South American Archaeology (5): 13-27.

Rojas Mora, S. 2008. Acerca de la complejidad social y sus referentes en el escenario del bajo río San Jorge (Caribe colombiano). Boletín de Antropología 22(39): 271-294.

Rojas Mora, S. 2010. Análisis espacial y patrones de asentamiento en el bajo río San Jorge (Caribe colombiano). Boletín de Antropología 24(41): 283-305.

Rojas Mora, S.; Montejo Gaitán, F. 2006. Manejo del espacio y aprovechamiento de recursos en la depresión Momposina. In: Valdez, F. Agricultura ancestral. Camellones y albarradas: contexto social, usos y retos del presente. Banco Central del Ecuador, Quito, 81-91.

Sanders, W.T.; Webster, D. 1978. Unilinealism, multilinealism, and the evolution of complex societies. In: Redman, C.L. et al. (Eds.). Social archeology: beyond subsistence and dating. Academic Press, New York, 249-302.

Sáenz Samper, J.; Lleras Pérez, R. 1999. Las relaciones prehispánicas entre los territorios de Costa Rica y Colombia. In: Oro y jade: emblemas de poder en Costa Rica. Museos Banco Central de Costa Rica: Museo Nacional de Costa Rica, San José; Museo del Oro, Bogotá, 16-37.

Sánchez Herrera, L.A.; Cooke, R.G. 1997. ¿Quién presta y quién imita?: orfebrería e iconografía en "Gran Coclé" Panamá. Boletín Museo del Oro (42): 87-111.

Scott Raymond, J. 1998. Beginnings of sedentism in the lowlands of Northwestern South America. In: Oyuela-Caycedo, A.; Scott Raymond, 
Más allá de peregrinos y de oro: desarrollo social precolombino Costa Rica, Panamá y el norte de Colombia

R. Museu Arq. Etn., 31: 56-79, 2018.

J. Recent advances in the archaeology of the Northern Andes. Cotsen Institute of Archaelogy, Los Angeles, 11-20.

Scott Raymond, J. 2008. The process of sedentism in northwestern South America. In: Silverman, H.; Isbell, W. Handbook of South American archaeology. Springer, New York, 79-90.

Sheets, P.D. 1992. The pervasive pejorative in Intermediate Area studies. In: Lange, F.W. (Ed.) Wealth and hierarchy in the Intermediate Area. Dumbarton Oaks Research Library and Collection, Washington, 15-41.

Sheets, P.D. 1994. Summary and conclusions. In: Sheets, P.D; Mckee, B.R. Archaeology, volcanism, and remote sensing in the Arenal region, Costa Rica. University of Texas Press, Texas, 312-326.

Serje de la Ossa, M. 1984. Organización urbana en Ciudad Perdida. Cuadernos de Arquitectura Escala (9): 1-22.

Serje de la Ossa, M. 1987. Arquitectura y urbanismo en la cultura Tairona. Boletín Museo del Oro (19): 87-96.

Smith, M.E. (Ed.). 2011. The comparative archaeology of complex societies. Cambridge University Press, Cambridge.

Snarskis, M.J. 1977. Turrialba (9-FG-T), un sitio paleoindio en el este de Costa Rica. Vínculos 3 (1-2): 13-26.

Snarskis, M.J. 1978. The Archaeology of the Central Atlantic Watershed of Costa Rica. Tesis de doctorado. Columbia University, New York.

Snarskis, M.J. 1979. Turrialba: a Paleo-Indian quarry and workshop site in eastern Costa Rica. American Antiquity 44(1): 125-138.

Snarskis, M.J. 1981. Archaeology of Costa Rica. In: Elizabeth, P.B. (Ed.). Between continents/between seas: precolumbian art of Costa Rica. Harry N. Abrams, New York, 15-84.
Snarskis, M.J. 1984. Central America: The lower Caribbean. In: Lange, F.W.; Stone, D.Z. The archaeology of Lower Central America. University of New Mexico Press, Albuquerque, 195-232.

Snarskis, M.J. 1986. Un modelo de la evolución cultural en Costa Rica (500 a.C.-1500 d.C.). In: Barrantes, R.; Bozzoli, M.E.; Gudiño, P. Memorias del primer simposio científico sobre pueblos indígenas de Costa Rica. Instituto Geográfico de Costa Rica, San José, 111-116.

Snarskis, M.J. 2003. From jade to gold in Costa Rica: how, why, and when. In: Quilter, J.; Hoopes, J.W. Gold and power in ancient Costa Rica, Panama, and Colombia. Dumbarton Oaks Research Library and Collection, Washington, 159-204.

Sol Castillo, R.F. 2013. Religious organization and political structure in prehispanic southern Costa Rica. Tesis de doctorado, University of Pittsburgh, Pittsburgh.

Taylor, W.W. 1948. A study of archaeology. American Anthropological Association, Washington.

Uribe Tobón, C.A. 1988. De la Sierra Nevada de Santa Marta, sus ecosistemas, indígenas y antropólogos. Revista de Antropología 4(1): 7-35.

Uribe Villegas, M.A. 1988. Introducción a la orfebrería de San Pedro de Urabá, una región del noroccidente colombiano. Boletín Museo del Oro (20): 35-53.

Uribe Villegas, M.A.; Martinón-Torres, M. 2014. Composition, colour and context in Muisca votive metalwork (Colombia, AD 600-1800). Antiquity 86(133): 772-791.

Valderrama Andrade, B.; Fonseca Truque, G. 1981. Exploraciones en la vertiente norte de la Sierra Nevada de Santa Marta. Boletín Museo del Oro (11): 1-41.

Van der Hammen, T.; Correal, G. 2001. Mastodontes en un humedal pleistocénico en el valle del Magdalena (Colombia) con evidencias de la 
presencia del hombre en el pleniglacial. Boletín de Arqueología 16(1): 4-36.

Willey, G.R. 1971. An introduction to American Archaeology. vol. 2: South America. Englewood Cliffs, New Jersey.
Wobst, H.M. 1977. Stylistic behavior and information exchange. In: Cleland, C. For the director; research essays in honor of James $B$. Griffin. University of Michigan Press, Ann Arbor, 317-343. 